Research Article

\title{
Green Synthesis of [EMIm]Ac Ionic Liquid for Plasticizing MC-based Biopolymer Electrolyte Membranes
}

\author{
Sun Theo Constan Lotebulo Ndruru ${ }^{*}$, Deana Wahyuningrum², Bunbun Bundjali1, \\ I Made Arcana ${ }^{1}$
}

\author{
${ }^{1}$ Inorganic and Physical Chemistry Division, Faculty of Mathematics and Natural Sciences, Institut \\ Teknologi Bandung, Jl. Ganesha 10, Bandung 40132, Indonesia \\ ${ }^{2}$ Organic Chemistry Division, Faculty of Mathematics and Natural Sciences, Institut Teknologi Bandung, \\ Jl. Ganesha 10, Bandung 40132, Indonesia
}

Received: $13^{\text {rd }}$ August 2018; Revised: 14th January 2019; Accepted: 1'st February 2019; Available online: 30th April 2019; Published regularly: 1st August 2019

\begin{abstract}
Lithium-ion batteries (LIBs) are favorable power source devices at the last two decades, owing to high energy density, rechargeable, long life cycle, portable, safe, rechargeable, good performance and friendly environment. To support their development, in this research has been successfully prepared polymer electrolyte membrane, a main component of LIBs, based on 1-ethyl-3-methylimidazolium acetate ([EMIm]Ac) ionic liquid-plasticized methyl cellulose/lithium perchlorate $\left(\mathrm{MC}_{\mathrm{LiClO}}\right)$. [EMIm] Ac ionic liquid was easy synthesized by metathesis reaction between 1-ethyl-3-methylimidazolium bromide $([\mathrm{EMIm}] \mathrm{Br})$ ionic liquid and potassium acetate $\left(\mathrm{CH}_{3} \mathrm{COOK}\right)$ at ambient temperature, for 1 hour. [EMIm]Ac ionic liquid was functional groups analyzed with Fourier Transform Infra-red (FT-IR) and structural analyzed with ${ }^{1} \mathrm{H}-\mathrm{Nuclear}$ Magnetic Resonance (NMR) and ${ }^{13} \mathrm{C}-\mathrm{NMR}$. [EMIm]Ac ionic liquidplasticized $\mathrm{MC} / \mathrm{LiClO}_{4}$ biopolymer electrolyte membrane was prepared by casting solution, with [EMIm]Ac ionic liquid content, 0, 5, 10, 15, 20, 25, and 30\% (w/w). Effect of $15 \%$ (w/w) [EMIm]Ac ionic liquid incorporation to $\mathrm{MC} / \mathrm{LiClO}_{4}$ showed the best condition and selected as the optimum condition with conductivity, tensile strength, elongation break, and thermal stability of $9.160 \times 10^{-3} \mathrm{~S}^{-\mathrm{cm}^{-1}, 24.19}$ $\mathrm{MPa}, 36.43 \%, \sim 256$ and $\sim 370{ }^{\circ} \mathrm{C}$, respectively. These results confirm that [EMIm]Ac ionic liquid can

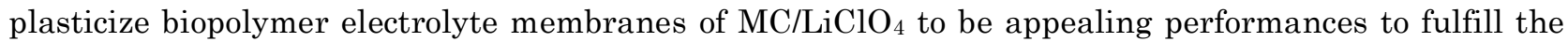
LIB's separator requirement. Copyright (C) 2019 BCREC Group. All rights reserved
\end{abstract}

Keywords: Ionic Liquids; Cellulose; Methyl cellulose; Lithium Ion Batteries

How to Cite: Ndruru, S.T.C.L., Wahyuningrum, D., Bundjali, B., Arcana, I.M. (2019). Green Synthesis of [EMIm]Ac Ionic Liquid for Plasticizing MC-based Biopolymer Electrolyte Membranes. Bulletin of Chemical Reaction Engineering \& Catalysis, 14(2): 345-357 (doi:10.9767/bcrec.14.2.3074.345-357)

Permalink/DOI: https://doi.org/10.9767/bcrec.14.2.3074.345-357

\section{Introduction}

Li-ion battery (LIB), nowadays, is a very important energy storage technology in human be-

\footnotetext{
* Corresponding Author.

E-mail :arcana@chem.itb.ac.id (I.M. Arcana); stcln07chem@gmail.com (S.T.C.L. Ndruru);

Telp. : +62-81-22381355
}

ing activities [1-2]. Lithium ion batteries (LIBs) are used extensively in many applications, such as: laptop computers, mobile phones, and others, due to their high energy density, long life time, and rechargeable [3-4]. However, some issues in the development of LiBs are the cost production, environment, safety, and performances. The issues are mainly related both electrolytes and separator [5]. 
Liquid electrolytes are corrosive and volatile cause leakage and explosion at high temperatures [6]. A thermally unstable separator material else tends to shrinkage at high temperatures causes memory effect. Solid polymer electrolytes (SPEs) are those macromolecules conducting ions and separating both cathode and anode electrodes [7]. However, SPEs have problem with their ionic conductivity which are lower than liquid electrolytes [8]. Some efforts to improve the ionic conductivity, they are introducing plasticizer (filler or ionic liquids), salts system mixtures, polymer blend and solvent system mixtures [9-10]. Porous SPEs are desirable due to exhibit advantages of liquid electrolyte (excellent ionic conductivity) and solid polymer electrolyte (outstanding mechanical property) [11]. It can be stated that the solid polymer electrolytes function as liquid electrolytes and common separator [12].

Applying the environmentally friendly and renewable materials overcome relatedproblems of LIBs. Cellulose is the most abundant biopolymer in nature and is widely used in many industrial uses, such as: paper making, food packaging, pharmaceutical applications and innovative eco-friendly application. Cellulose derivatives have been successfully applied as polymer electrolytes for LIBs separator application, they are, methyl cellulose [6,13], ethyl cellulose [14], hydroxyethyl cellulose [2], carboxymethyl cellulose [15-16].

Methyl cellulose (MC), an important ether cellulose, is widely used owing to have many advantages, they are, biocompatible, inexpensive, ease to make thin films and edible films. It also has outstanding mechanical properties compared to other biodegradable polymers such as chitosan, but it is recommended to increase ion conductivity [17].

Ionic liquids are a distinctive and interesting material due to have appealing properties. The properties are high boiling point, nonvolatile, good conductivity, good thermal stability, plastic effect, wide electrochemical potential, reusable, and reproduceable [18]. These attractive properties are widely used in various purposes, such as cellulose dissolution [19-20], synthesis, electrolytes [21], as plasticizers [3,13,22], and corrosion inhibitors [23].

Ionic liquids consist of organic cations (bulky) and organic/inorganic anions (bulky / polyatom / halide). Weak ionic interactions, causing ionic liquids to easily be produced to new ionic liquid derivatives with different properties. Metathesis reaction is commonly method to derive new ionic liquids.
A microwave is one of electromagnetic energy that absorbs rotation energy of molecules with $300-300,000 \mathrm{MHz}$ of frequency. This principle is used in microwave assisted organic synthesis (MAOS). Microwave-assisted heating accelerates producing product with dipole rotation and ionic conduction [24]. MAOS method is promising to ionic liquids synthesis. Both microwave heating and ionic liquids are the "hot topics" in chemistry [25]. Ionic liquids synthesis, generally, can be described in two steps, they are the desired cation formation and anion exchange (metathesis reaction) [26].

Some advantages of microwave heating utilization, practically, are mainly shorter time duration of reaction, compare to conventional heating methods. Reaction time reducing of microwave irradiation is owing to extensive heating direct to reaction system without through the vessel (conventional heating methods) [27].

The effect of ionic liquid plasticization in solid polymer electrolyte membranes has been successfully studied. Some reports approved that there are significantly improved performances of LIBs due to ionic liquids incorporation in polymer electrolyte membranes. Several researches also have succeeded to study the effects of ionic liquids plasticization of LIBs performances [3,13,22]. The properties needed as electrolyte are negligible vapor point, large liquid-phase temperature interval, nonflammable, good thermal stability and elevated electrochemical stability [28-29].

The 1-ethyl-3-methylimidazolium acetate [EMIm]Ac is popular ionic liquid which able to dissolve and deconstruct cellulose, besides 1-butyl-3-mehylimimidazolium chloride ([BMIm]Cl) [30] and 1-ally-3methylimidazolium chloride [AMIm]Cl [31]. However, they have never been reported to be electrolytes and/or plasticizers. Only few reports used $[\mathrm{BMIm}] \mathrm{Cl}$ ionic liquid in polymer electrolytes study. The potential of [EMIm]Ac ionic liquid to be electrolyte and/or plasticizer is attributed to electron withdrawing group $\left(\mathrm{CH}_{3} \mathrm{COO}^{-}\right)$which can interact to polymer chain, so it contributes ion migration.

This work investigated novel biopolymer electrolyte (BPE) membrane. The effect of ionic liquid plasticization, an environmentally friendly material, is compatible to methyl cellulose (MC)-based biopolymers. Ionic liquid was synthesized using a MAOS method, while BPE membrane was simply prepared with casting solution technique. Functional groups, structural studies, ionic conductivities, mechanical properties, crystallinities, and thermal stability were carried out with Fourier 
transform infrared (FTIR), nuclear magnetic resonance (NMR), electrochemical impedance spectroscopy (EIS), tensile strength test, X-ray diffraction (XRD), scanning electron microscopy (SEM), and thermogravimetry analysis (TGA), respectively.

\section{Materials and Methods}

\subsection{Materials}

All materials and solvents were used without further purification, they were bromoethane (Sigma-Aldrich, 98\%), 1-methylimidazole (Sigma-Aldrich, 99\%), potassium acetate $\left(\mathrm{CH}_{3} \mathrm{COOK}\right)$ (Sigma-Aldrich, 99\%), methyl cellulose (MC) (Sigma-Aldrich, MW = 88,000; DS = 1.5-1.9), lithium perchlorate $\left(\mathrm{LiClO}_{4}\right)$ (SigmaAldrich, 99\%), chloroform $\left(\mathrm{CHCl}_{3}\right)$ (Merck, 99\%), methanol $\left(\mathrm{CH}_{3} \mathrm{OH}\right)$ (Merck, 99\%), and distilled water (Merck, 99\%).

\subsection{Methods}

2.2.1 The 1-ethyl-3-methylimidazolium bromide [EMIm] Br ionic liquid synthesis

[EMIm]Br ionic liquid as a precursor was synthesis using Microwave Assisted Organic Synthesis (MAOS) method. The 1-methylimidazole and bromoethane were dissolved into chloroform, then stirred for 24 hours. The solution mixture was then subjected onto microwave reactor CEM. Microwave heating was carried out at power of 100 watt, $50{ }^{\circ} \mathrm{C}$, 15 minutes, and medium stirring. Highly purity of [EMIm]Br was obtained using extraction (water : ethyl acetate $=1: 1$ ). Water fraction, then, vacuum distilled to produce pure interested-[EMIm]Br ionic liquid. The purified [EMIm]Br ionic liquid was then FTIR and NMR characterized. The [EMIm]Br synthesis reaction scheme is shown in Figure 1.

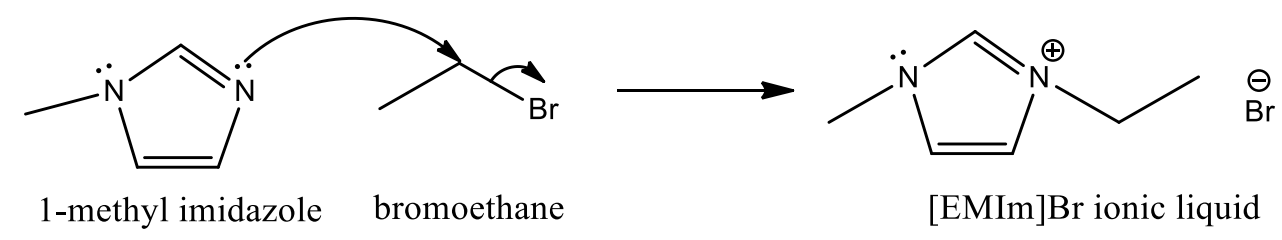

Figure 1. Reaction scheme of [EMIm]Br synthesis

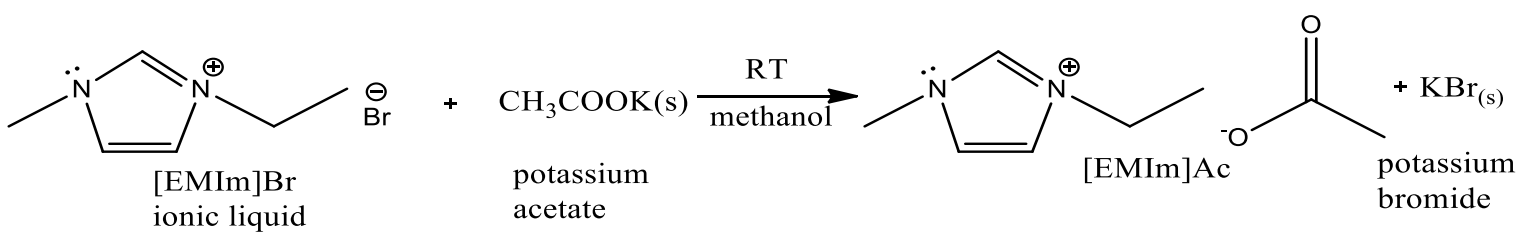

Figure 2. Reaction scheme of [EMIm]Ac ionic liquid synthesis

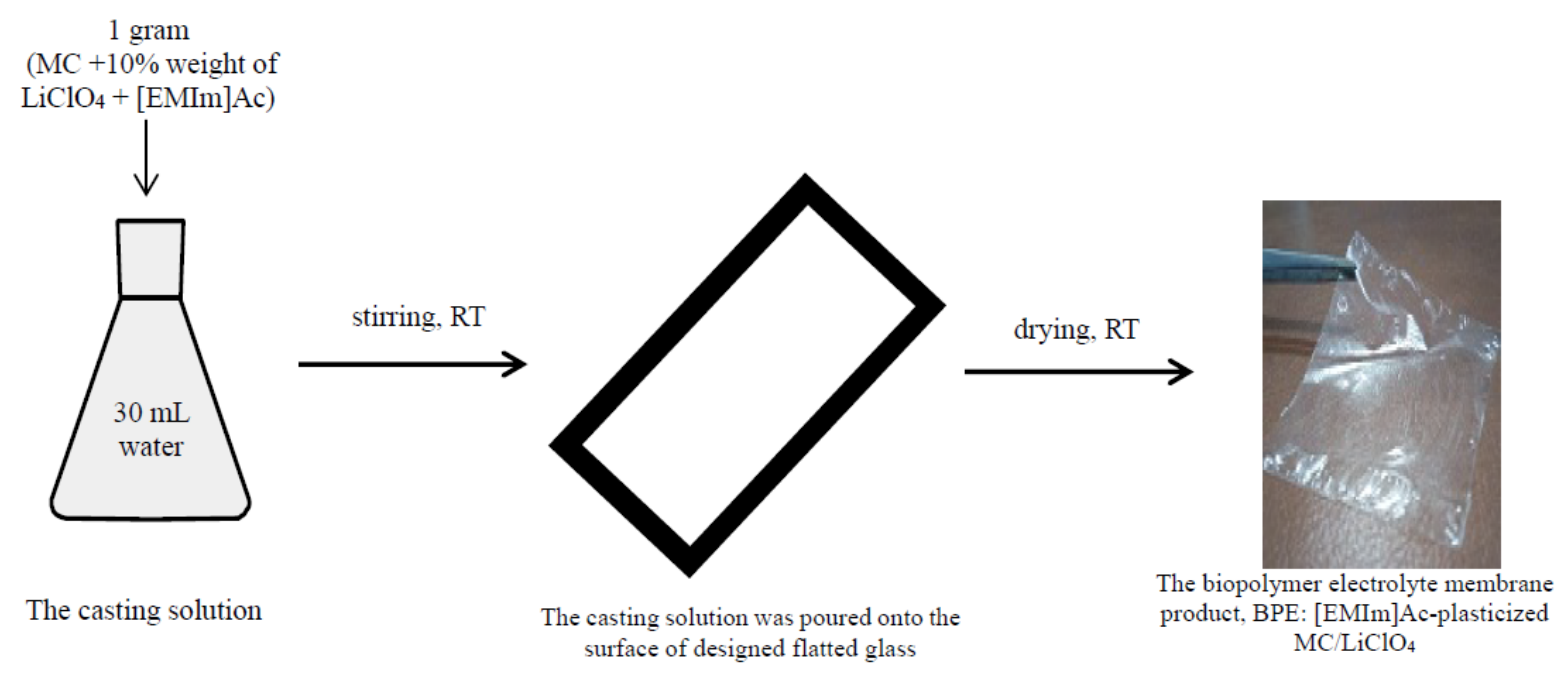

Figure 3. The preparation of biopolymer electrolyte membrane (BPE: \{EMIm]Ac-plasticized $\mathrm{MC} / \mathrm{LiClO}_{4}$ 
2.2.2 The 1-ethyl-3-methylimidazolium acetate ([EMIm] Ac) ionic liquid synthesis

[EMIm]Ac ionic liquid synthesis was employed with metathesis reaction between 0.2 mole previous produced [EMIm] $\mathrm{Br}$ ionic liquid and 0.2 mole $\mathrm{CH}_{3} \mathrm{COOK}$ salt in methanol. The released potassium bromide $(\mathrm{KBr})$ solid as byproduct was decanted from solution phase, was then evaporated by using vacuum evaporator at $50-60{ }^{\circ} \mathrm{C}$ to remove methanol solvent. The salt solid was [EMIm]Ac ionic liquid, that was then FTIR and NMR characterized. The metathesis scheme of the [EMIm]Br ionic liquid and $\mathrm{CH}_{3} \mathrm{COOK}$ metathesis to produce the [EMIm]Ac ionic liquid is shown in Figure 2.

\subsubsection{Biopolymer electrolyte membranes prepa-} ration

Biopolymer electrolyte membranes, BPEMs: $\mathrm{MC} / \mathrm{LiClO}_{4} /[\mathrm{EMIm}] \mathrm{Ac}$ were prepared using the casting solution technique (Figure 3). Total weight of prepared material was 1 gram dissolved in $30 \mathrm{~mL}$ of distilled water. Casting solution was then cast on the surface of the flatted glass, then evaporated at ambient temperature, continued drying in the vacuum oven. Complex of $\mathrm{MC} / \mathrm{LiClO}_{4}$ successfully prepared were $\mathrm{MC} / 10 \%$ wt $\mathrm{LiClO}_{4} / x$ [EMIm] Ac ionic liquid $(x=$ $5,10,15,20,25$, and $30 \%$ weight).

\subsection{Characterizations}

Complexation study of complexed-systems of biopolymer electrolytes membranes were carried out with FT-IR (Prestige 21 Shimadzu), ionic conductivities by electrochemical impedance spectroscopy (EIS, Agilent, type E490A, precision LCR), mechanical properties by tensile tester (FAVIGRAPH), crystallinity by diffractometer XRD (Rigaku Smart Lab) and surface morphology by Scanning electromotive morphology (SEM) (Type JEOL-JSM-6510LV), and thermal properties of biopolymer electrolytes membranes were measured by thermal

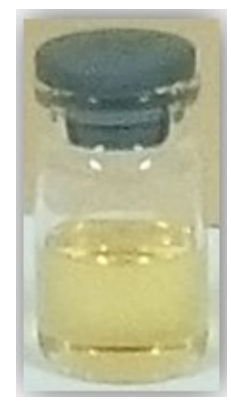

Figure 4. [EMIm] Br ionic liquid product gravimetry analysis (TGA, Linseis, type STA PT 1600).

\section{Results and Discussion}

\subsection{Synthesis of [EMIm]Br Ionic Liquid}

The 1-ethyl-3-methylimidazolium bromide ([EMIm]Br) ionic liquid was synthesized using Microwave Assisted Organic Synthesis (MAOS) method (in this research using CEM microwave reactor). The reagents used to [EMIm] Br ionic liquid synthesis were 1-methylimidazole and bromoethane $(1: 1)$. Setting the microwave reactor to synthesize [EMIm] Br ionic liquid were 100 watts, 15 minutes and $50{ }^{\circ} \mathrm{C}$. The reaction involved the substitution scheme of $\mathrm{S}_{\mathrm{N}} 2$. The free electron pair of 1-methylimidazole acts as a nucleophile that attacks the bromoethane which acts as an electrophile while releasing the bromide ion as a good leaving group.

The synthesis product [EMIm]Br ionic liquid is yellowish liquid. The product of [EMIm]Br synthesis was then washed by extraction: water/ ethyl acetate 3 times. The lower phase ([EMIm]Br) was separated, and then distilled by vacuum distillation. The purified [EMIm]Br ionic liquid has density of $1.8 \mathrm{~g} / \mathrm{mL}$ (Figure 4).

[EMIm]Br ionic liquid synthesis using MAOS method fulfills what green chemistry principle is. They are especially to prevention, less hazardous chemical synthesis, designing safer chemicals and design for energy efficiency [30].

3.2 FTIR and NMR Analysis of [EMIm]Br Ionic Liquid

The result of synthesis of 1-ethyl-3methylimidazolium bromide ([EMIm]Br) ionic liquid precursor identified functional group us-

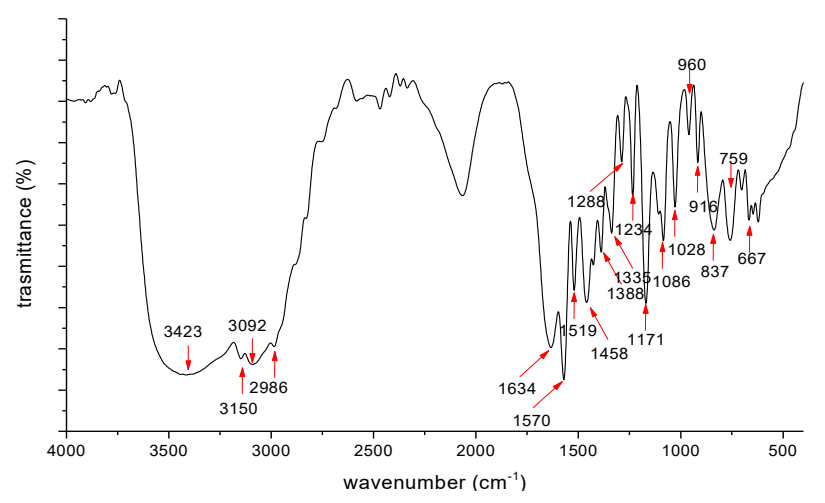

Figure 5. FTIR spectrum of [EMIm]Br ionic liquid 
ing Fourier Transform Infra-red (FT-IR) and its peak absorption analysis can be seen in Figure 5 . According to the Figure 5, several vibration modes for the [EMIm]Br ionic liquid are: $\mathrm{O}-\mathrm{H}$ stretching at $3423 \mathrm{~cm}^{-1},=\mathrm{C}-\mathrm{H}$ stretching of imidazole ring at $3150 \mathrm{~cm}^{-1}$ and $3092 \mathrm{~cm}^{-1}, \mathrm{C}-\mathrm{H}$ aliphatic stretching at $2986 \mathrm{~cm}^{-1}, \mathrm{C}=\mathrm{C}$ stretching of imidazole ring at $1635 \mathrm{~cm}^{-1}, \mathrm{C}=\mathrm{N}$ stretching of imidazole ring at $1570 \mathrm{~cm}^{-1}, \mathrm{C}-\mathrm{H}\left(\mathrm{CH}_{3}\right)$ bending at 1388 and $1335 \mathrm{~cm}^{-1}, \mathrm{C}-\mathrm{N}$ stretching imidazole at $1171 \mathrm{~cm}^{-1}, \mathrm{C}-\mathrm{N}$ interaction of $\mathrm{CH}_{3}$ and $\mathrm{N}$ of imidazole at $1086 \mathrm{~cm}^{-1},=\mathrm{C}-\mathrm{H}$ out of plane of imidazole ring at $837 \mathrm{~cm}^{-1}$ and $759 \mathrm{~cm}^{-1}$.

To ensure the [EMIm]Br ionic liquid structure, ${ }^{1} \mathrm{H}-\mathrm{NMR}$ and ${ }^{13} \mathrm{C}-\mathrm{NMR}$ analysis

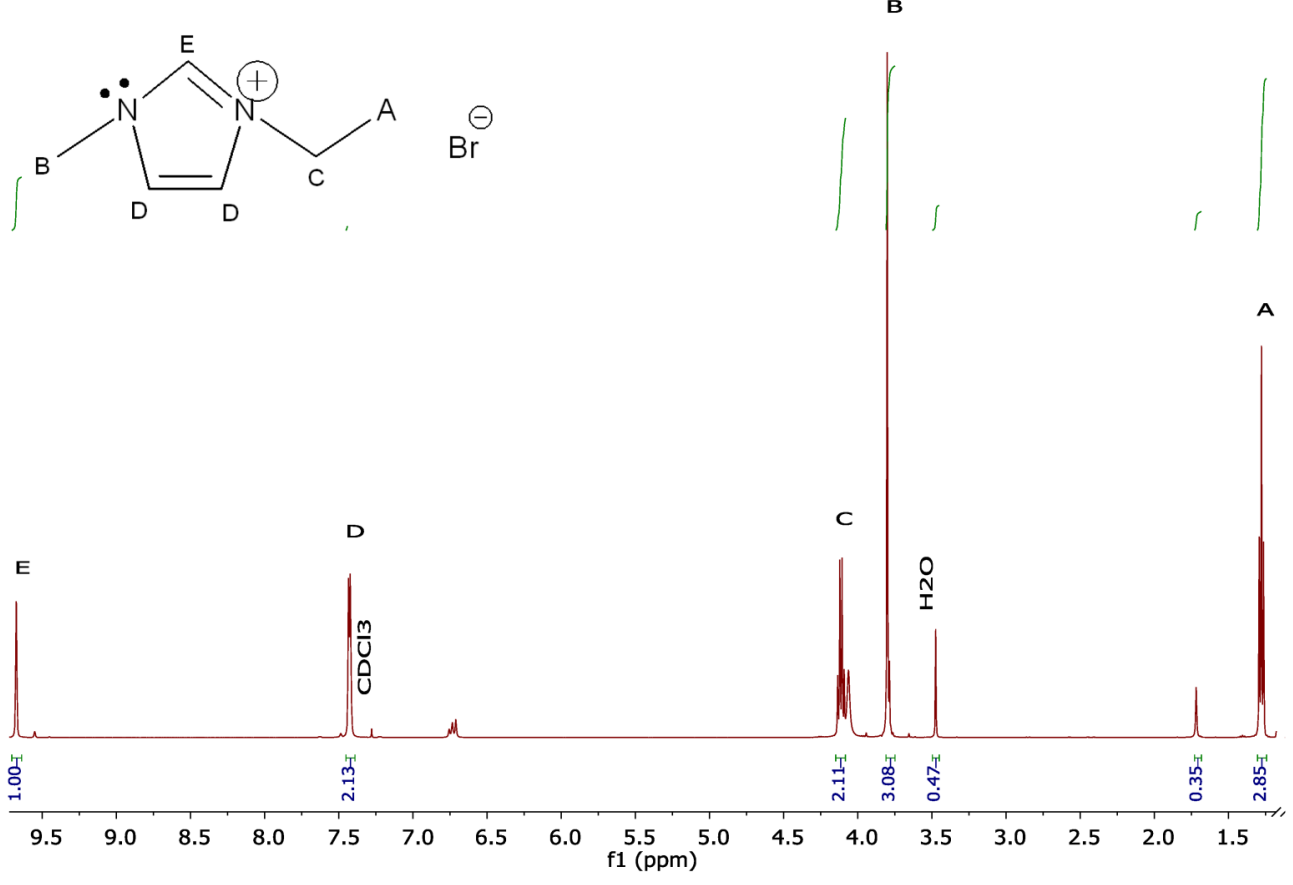

Figure 6. ${ }^{1} \mathrm{H}-\mathrm{NMR}$ spectrum of [EMIm]Br ionic liquid<smiles>[B]n1cc[n+](CC)c1</smiles>

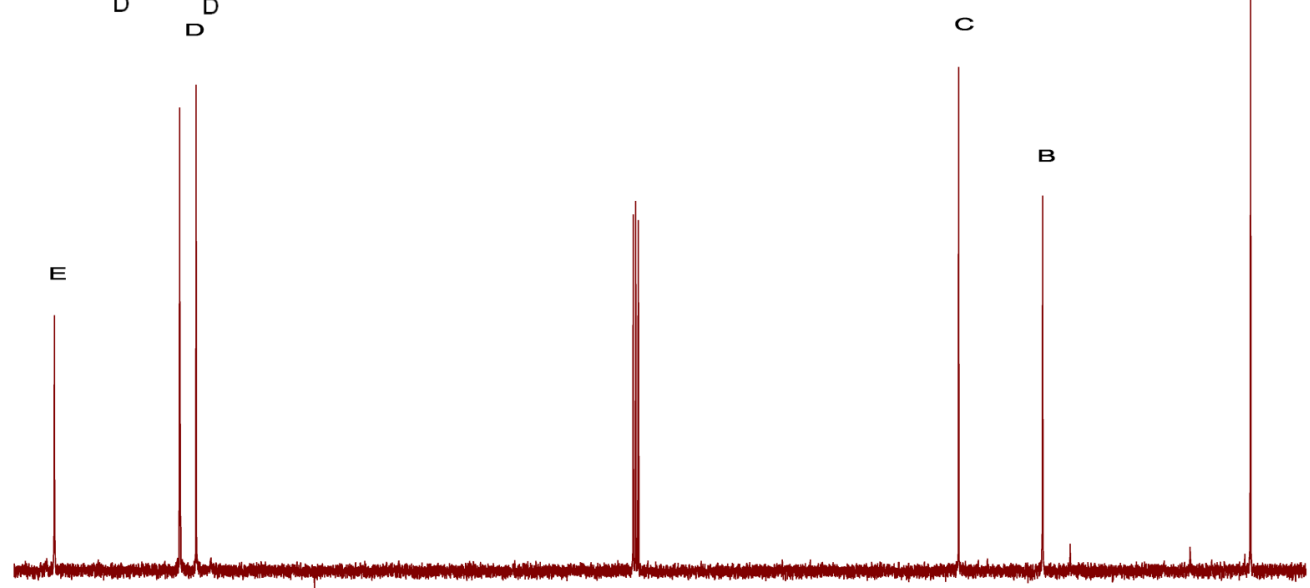

$\begin{array}{llllllllllllllllllllllllllllllllllllll}40 & 135 & 130 & 125 & 120 & 115 & 110 & 105 & 100 & 95 & 90 & 85 & 80 & 75 & 70 & 65 & 60 & 55 & 50 & 45 & 40 & 35 & 30 & 25 & 20 & 15 & 1\end{array}$

Figure 7. ${ }^{13} \mathrm{C}-\mathrm{NMR}$ spectrum of [EMIm]Br ionic liquid 
was performed. The ${ }^{1} \mathrm{H}-\mathrm{NMR}$ and ${ }^{13} \mathrm{C}-\mathrm{NMR}$ spectra are shown in Figure 6 and Figure 7. The results of the ${ }^{1} \mathrm{H}-\mathrm{NMR}$ and ${ }^{13} \mathrm{C}-\mathrm{NMR}$ analysis of [EMIm]Br ionic liquid are as follows:

${ }^{1} \mathrm{H}-\mathrm{NMR} \quad\left(500 \mathrm{MHz}, \mathrm{CDCl}_{3}\right): \quad \delta \quad(\mathrm{ppm}) \quad 1.28$ $(\mathrm{A}, 3 \mathrm{H}, t) ; 3.80(\mathrm{~B}, 3 \mathrm{H}, s) ; 4.11(\mathrm{C}, 2 \mathrm{H}, q) ; 7.44$ $(\mathrm{D}, 2 \mathrm{H}, d d) ; 9.68(\mathrm{E}, 1 \mathrm{H}, s)$.

${ }^{13} \mathrm{C}$-NMR (500 MHz, $\left.\mathrm{CDCl}_{3}\right): \delta(\mathrm{ppm}) 15,48(\mathrm{~A})$; 36,47 (B); 44,95 (C); 123,01 (D); 136,26 (E).

Based on ${ }^{1} \mathrm{H}-\mathrm{NMR}$ and ${ }^{13} \mathrm{C}-\mathrm{NMR}$ spectrum analysis, it confirms that [EMIm] Br ionic liquid were already formed from 1-methylimidazole and bromoethane precursors.

\subsection{Synthesis of [EMIm]Ac Ionic Liquid}

Synthesis of [EMIm]Ac ionic liquid was employed by simply metathesis reaction by mixing 0.2 mole of [EMIm] Br ionic liquid and 0.2 mole of potassium acetate $\left(\mathrm{CH}_{3} \mathrm{COOK}\right)$ in methanol. The potassium bromide (KBr) by-product was separated from solution. The solution was evaporated by vacuum evaporator to remove methanol solvent, released yellowish [EMIm]Ac salt solid (Figure 8). Similar to [EMIm]Br ionic liquid synthesis, so [EMIm]Ac ionic liquid synthesis also fulfill the green chemistry principle. It's especially to process performing at ambient temperature and pressure [30].

3.4 FT-IR dan NMR Analysis of [EMIm]Ac Ionic Liquid

The [EMIm]Ac ionic liquid was analyzed by FT-IR as shown in Figure 9. According to the spectrum, several vibration modes for the [EMIm]Ac ionic liquid are shown: $\mathrm{O}-\mathrm{H}$ stretching at $3468 \mathrm{~cm}^{-1},=\mathrm{C}-\mathrm{H}$ stretching of imidazole ring at $2983 \mathrm{~cm}^{-1}, \mathrm{C}-\mathrm{H}$ aliphatic stretching at $2822 \mathrm{~cm}^{-1}, \mathrm{C}=\mathrm{C}$ stretching of imidazole ring is not visible, while the $\mathrm{C}=\mathrm{N}$ stretching of imidazole ring is broad at $1597 \mathrm{~cm}^{-1}, \mathrm{O}-\mathrm{H}$ bending is strong at 1408 and $920 \mathrm{~cm}^{-1}$, is seen at $1338 \mathrm{~cm}^{-}$ 1 , which also still indicates a bending vibration

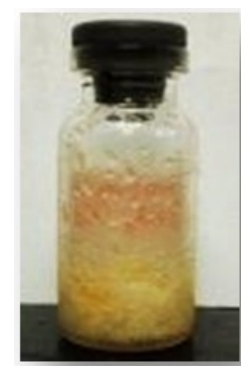

Figure 8. Product of [EMIm]Ac ionic liquid of $\mathrm{C}-\mathrm{H}\left(\mathrm{CH}_{3}\right)$ in the region of the wave number, $\mathrm{C}-\mathrm{N}$ stretching of imidazole ring at $1172 \mathrm{~cm}^{-1}$, $\mathrm{C}-\mathrm{N}$ interaction of $\mathrm{CH}_{3}$ and $\mathrm{N}$ of imidazole ring at $1032 \mathrm{~cm}^{-1},=\mathrm{C}-\mathrm{H}$ out of plane of imidazole ring at $756 \mathrm{~cm}^{-1}$.

The substituted-bromide ion with acetate ion $\left(\mathrm{CH}_{3} \mathrm{COO}^{-}\right)$was confirmed according to the presence of a group with a strong intensity and broad at 3468, 15961419 and $1032 \mathrm{~cm}^{-1}$, as shown in Figure 9, assumed to interaction between the $\left[\mathrm{EMIm}^{+}\right]$cation and polar sites of polymer chain. The NMR analysis was performed to confirm [EMIm]Ac ionic liquid structure.

The results of the ${ }^{1} \mathrm{H}-\mathrm{NMR}$ and ${ }^{13} \mathrm{C}-\mathrm{NMR}$ analysis of [EMIm]Ac ionic liquid are as follows:

${ }^{1} \mathrm{H}-\mathrm{NMR}\left(500 \mathrm{MHz}, \mathrm{CDCl}_{3}\right): \delta$ (ppm) 1.53-1.56 (A, 3H, t); 3.95-3.96 (B, 3H, s); 4.27-4.31 (C, $2 \mathrm{H}$, $m) ; 7.60-7.68(\mathrm{D}, 2 \mathrm{H}, d d) ; 9.02(\mathrm{E}, 1 \mathrm{H}, s) ; 1.90$ $(\mathrm{F}, 3 \mathrm{H}, s)$.

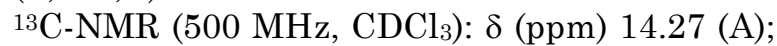
35.14 (B); 44.63 (C); 122.73 (D); 127.60 (E); $23.00(\mathrm{~F}) ; 178.81$ (G).

Based on the ${ }^{1} \mathrm{H}$-NMR and ${ }^{13} \mathrm{C}-\mathrm{NMR}$ spectrum analysis, it confirms that [EMIm]Ac ionic liquid were already formed from 1methylimidazole and bromoethane precursors.

3.5 FTIR Analysis and Complexation Study of Biopolymer Electrolyte (BPE) Membranes MC/ $\mathrm{LiClO}_{4} /[\mathrm{EMIm}] \mathrm{Ac}$

The biopolymer electrolyte membranes, BPE membranes: $\mathrm{MC} / \mathrm{LiClO}_{4} /[\mathrm{EMIm}] \mathrm{Ac}$ produced in the form of transparent thin film, with average thickness of $\sim 22 \mu \mathrm{m}$, meets the minimum requirements of LIB's separator application $(\sim 100 \mu \mathrm{m})$. The FTIR spectrum of the biopolymer electrolyte membranes, BPE membranes: $\mathrm{MC} / \mathrm{LiClO}_{4} /[\mathrm{EMIm}] \mathrm{Ac}$ is shown in Figure 12.

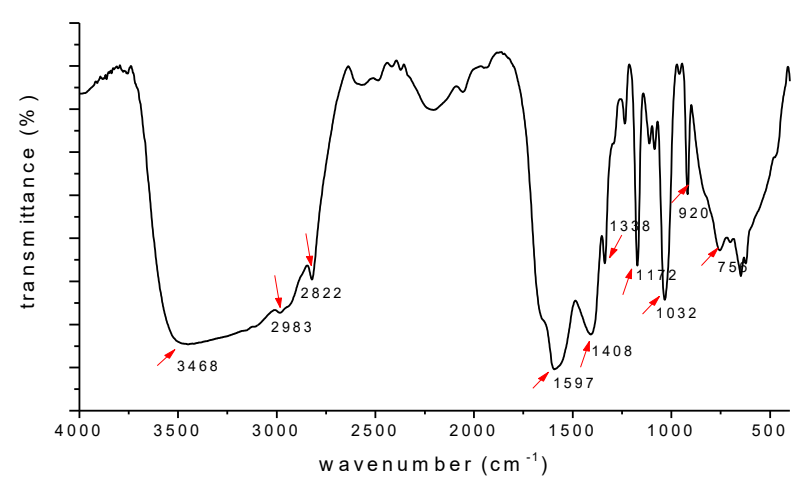

Figure 9. FTIR spectrum of [EMIm]Ac ionic liquid 
<smiles>[3H]C1CCC(C)[N+]1=C</smiles><smiles>O=C(O)F</smiles>
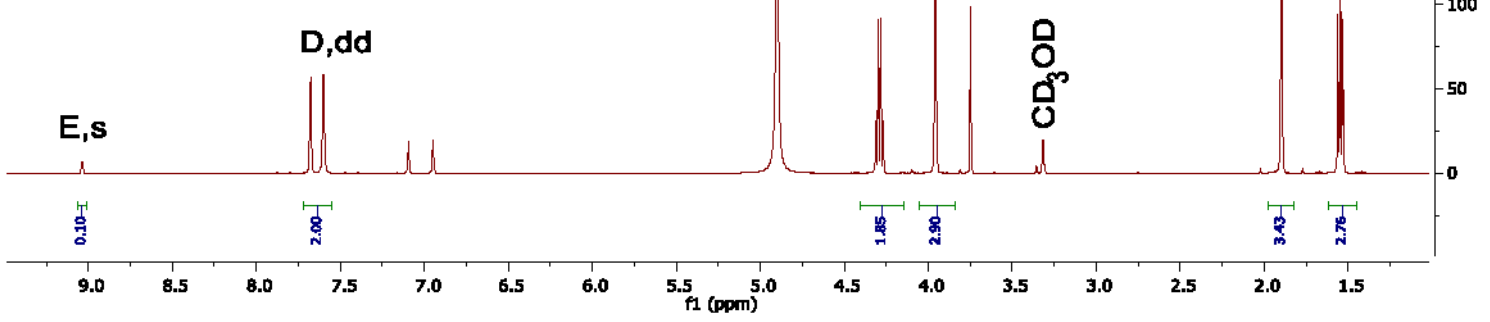

Figure 10. ${ }^{1} \mathrm{H}-\mathrm{NMR}$ spectrum of $[\mathrm{EMIm}] \mathrm{Ac}$ ionic liquid<smiles></smiles><smiles>O=C(O)F</smiles>

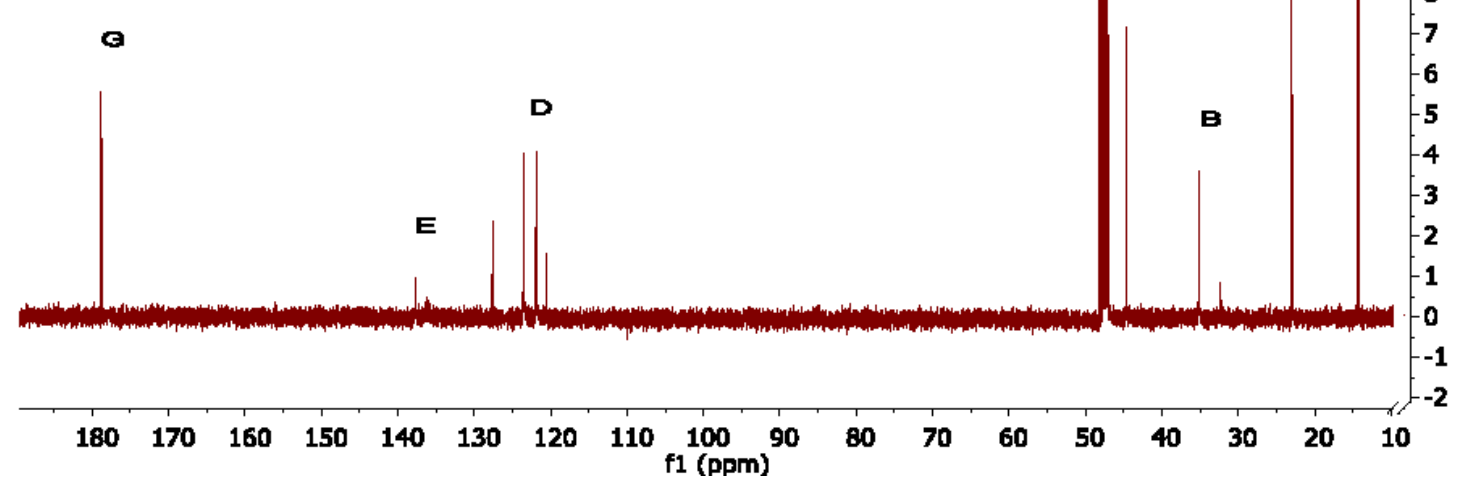

Figure 11. ${ }^{13} \mathrm{C}-\mathrm{NMR}$ spectrum of [EMIm] Ac ionic liquid 
The broad H-O-H bending vibration of methyl cellulose around $1645 \mathrm{~cm}^{-1}$, is gradually reduced as an increase of [EMIm]Ac ionic liquid concentration. The incorporation of $15 \%$ to $30 \%$ $(\mathrm{w} / \mathrm{w})$ of the $[\mathrm{EMIm}] \mathrm{Ac}$ ionic liquid to $\mathrm{LiClO}_{4-}$ complexed $\mathrm{MC}$ reduces the typical peak of $\mathrm{MC} / \mathrm{LiClO}_{4}$, while increasing the new absorption peak at $1570 \mathrm{~cm}^{-1}$, identified as an aromatic $\mathrm{C}-\mathrm{C}$ group of [EMIm]Ac ionic liquid.

In the previous FT-IR spectrum of [EMIm]Ac ionic liquid shown two typical $\mathrm{C}-\mathrm{C}$ peaks absorption of imidazolium cations, could be seen at 1593.20 and $1408.04 \mathrm{~cm}^{-1}$. Based on previous reports [32], the absorption bands at 1593.20 and $1408.04 \mathrm{~cm}^{-1}$ are asymmetry and symmetry $\mathrm{COO}^{-}$group. Thus, based on this FTIR study, C-C absorption of aromatic ring and COO-group overlap each other on the same wave number. The intensity is getting stronger at $1570 \mathrm{~cm}^{-1}$, along with \% weight of $5-30 \%$ $(\mathrm{w} / \mathrm{w})$ [EMIm]Ac ionic liquid to $\mathrm{LiClO}_{4-}$ complexed MC.

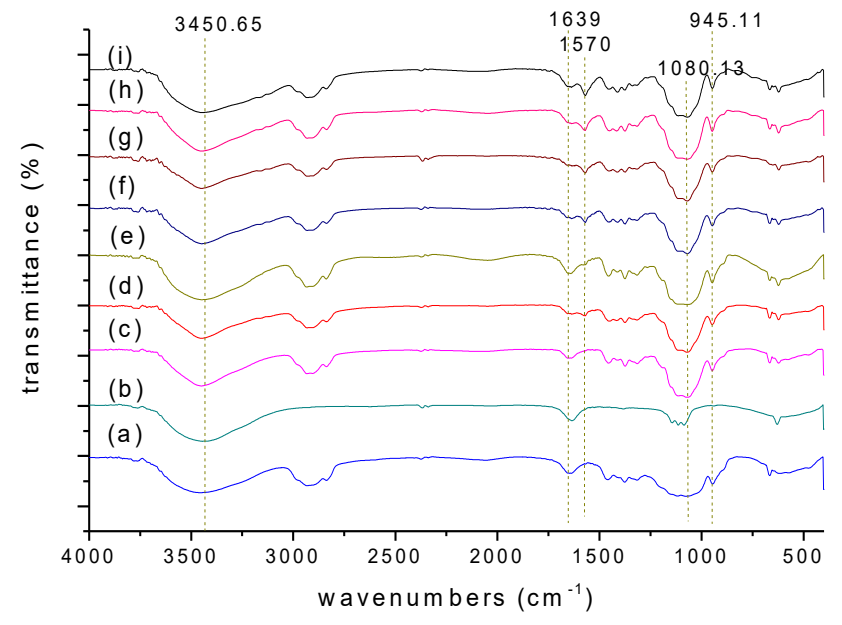

Figure 12. FTIR spectra (a) Pure MC, (b) $\mathrm{LiClO}_{4}$, (c) $\mathrm{MC} / 10 \%$ (w/w) $\mathrm{LiClO}_{4}$, and $\mathrm{MC} / 10 \%$ $(\mathrm{w} / \mathrm{w}) \mathrm{LiClO}_{4} / x \%$ [EMIm]Ac, $x(\% \mathrm{w} / \mathrm{w}):$ (d) 5 , (e) 10, (f) 15 , (g) 20 , (h) 25, (i) 30
The [EMIm]Ac ionic liquid incorporation to $\mathrm{MC} / \mathrm{LiClO}_{4}$ matrix biopolymer electrolytes causes some important peaks of $\mathrm{MC} / \mathrm{LiClO}_{4}$ are shift and even disappeared. These are due to complexation or interaction between the ether oxygen of $\mathrm{MC}$ and cations $\left(\left[\mathrm{EMIm}^{+}\right]\right.$and $\mathrm{Li}^{+}$). The shifting and intensity reducing of several peaks absorption are mainly at hydrogen bond stretching and bending, as interacting between [EMIm]Ac ionic liquid and $\mathrm{LiCO}_{4}$-complexed methyl cellulose. The geometry complexation of $\mathrm{MC}$ hydroxyl with [EMIm+] and $\mathrm{Li}^{+}$is shown in Figure 13.

3.6 Ionic Conductivities Biopolymer-Electrolyte Membranes, BPE Membranes: $\mathrm{MC} / \mathrm{LiClO}_{4} /$ [EMIm]Ac

The ionic conductivities of the biopolymer electrolyte membranes, BPE membranes: $\mathrm{MC} / \mathrm{LiClO}_{4} /[\mathrm{EMIm}] \mathrm{Ac}$ at room temperature is shown in Table 1. The ionic conductivity of BPE membranes increases with increasing [EMIm]Ac ionic liquid concentration. [EMIm]Ac ionic liquid is fully contributing to ionic conductivity increasing, thanks to its functions as a spacer.

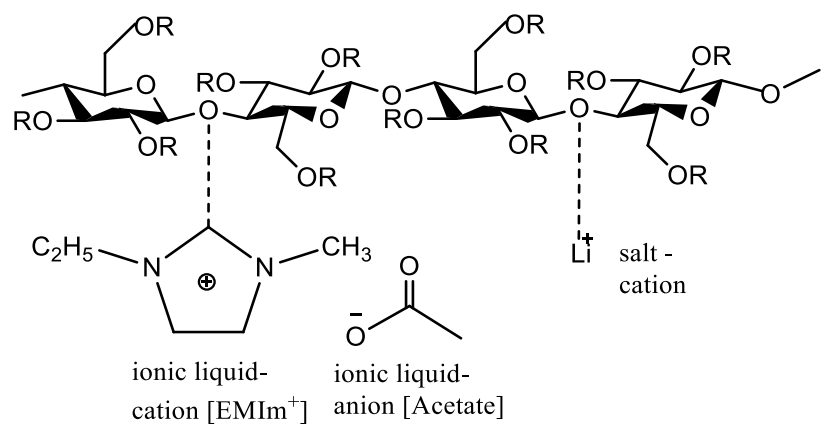

Figure 13. Proposed mechanism of complexation of MC hydroxyl with $\left[\mathrm{EMIm}^{+}\right]$and $\mathrm{Li}^{+}$ (adapted from previous report [29]

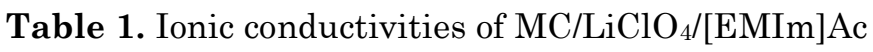

\begin{tabular}{cccc}
\hline $\begin{array}{c}\mathrm{MC} \text { content } \\
(\%)\end{array}$ & $\begin{array}{c}\mathrm{LiClO}_{4} \text { content } \\
(\%)\end{array}$ & {$[\mathrm{MIm}]$ Ac ionic liquid content (\%) } & $\begin{array}{c}\text { Ionic Conductivities, } \sigma \\
\left(\mathrm{S} . c m^{-1}\right)\end{array}$ \\
\hline 80 & 10 & 0 & $3.950 \times 10^{-5}$ \\
75 & 10 & 5 & $5.560 \times 10^{-3}$ \\
70 & 10 & 10 & $6.050 \times 10^{-3}$ \\
65 & 10 & 15 & $9.160 \times 10^{-3}$ \\
60 & 10 & 20 & $1.782 \times 10^{-2}$ \\
55 & 10 & 25 & $3.090 \times 10^{-3}$ \\
50 & 10 & 30 & $1.646 \times 10^{-3}$ \\
\hline
\end{tabular}


The highest conductivity is at $20 \%(\mathrm{w} / \mathrm{w})$ [EMIm]Ac ionic liquid incorporation of $1.782 \times 10^{-2} \mathrm{~S} . c m^{-1}$. After the composition, the ionic conductivities are gradually decreasing, and this is predicted due to an increasing of ionic liquid concentration providing a steric hindrance to $\mathrm{Li}^{+}$ion mobilization. $\mathrm{Li}^{+}$-ion mobilization decreases, as the interaction of the acetate ion dipole to $\mathrm{Li}^{+}$-ion and $\mathrm{MC}$ increases after the incorporation of ionic liquid under optimum conditions.

Decreased ionic conductivity is due to an increase in dipole interaction between ionic liquids and $\mathrm{MC} / \mathrm{LiClO}_{4}$ compounds. This reduction in conductivity is also thought to be caused by the aggregation of ions that reduce the amount of charge carriers and limit the movement of ions [32]. This reason was also previously reported [33], which said that the empirical formulas-associations by ions cause less chargecarrying motion and form larger ion clusters that limit ionic motion, thus causing ionic conductivity to decrease. In Table 1 . below, it is shown the value of biopolymer electrolyte membrane conductivities, MBE: $\mathrm{MC} / \mathrm{LiClO}_{4} /[\mathrm{EMIm}] \mathrm{Ac}$.

3.7 Mechanical Properties of BiopolymerElectrolyte Membranes, BPE Membranes: $\mathrm{MC} / \mathrm{LiClO}_{4} /[\mathrm{EMIm}] \mathrm{Ac}$

There are two analysis to study the mechanical properties of the BPE membranes: $\mathrm{MC} / \mathrm{LiClO}_{4} /[\mathrm{EMIm}] \mathrm{Ac}$ system, they are the tensile strength and elongation at break. In Figure 14 depicted that $\mathrm{LiClO}_{4}$-complexed methyl cellulose has high of tensile strength (stress), and continued by trend of effect of [EMIm]Ac ionic

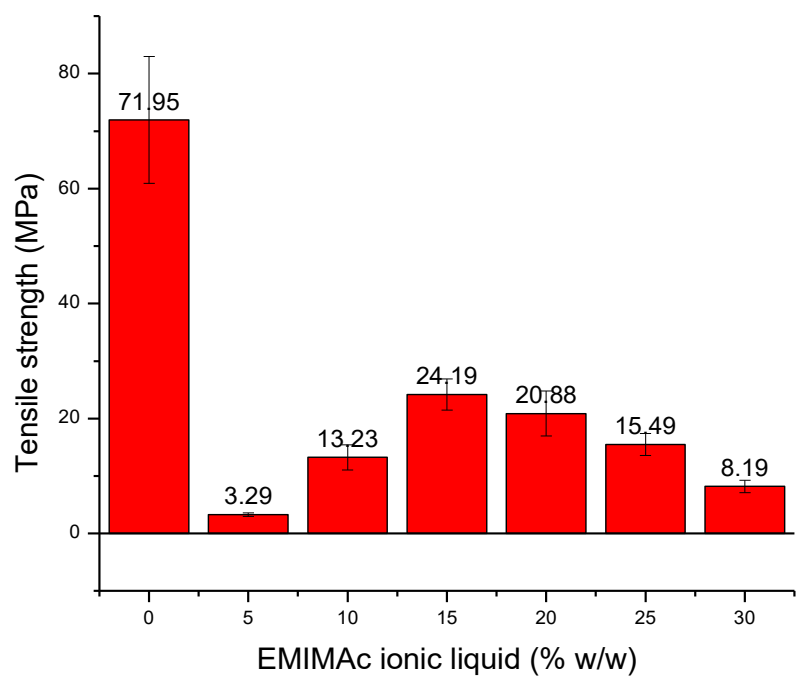

Figure 14. Tensile strength (stress) of BPE Membranes liquid incorporation to tensile strength deformation. [EMIm]Ac ionic liquid incorporation decreases tensile strength of BPE membranes initially, however, following incorporation improves this property gradually until optimum condition.

[EMIm]Ac ionic liquid was incorporated to BPE membranes with various contents, i.e 5, $10,15,20,25$, and $30 \%(\mathrm{w} / \mathrm{w})$. The [EMIm]Ac ionic liquid effect to BPE membranes tensile strength can be observed as shown in Figure 14. From the data, [EMIm]Ac ionic liquid causes tensile strength decreased drastically at its initial incorporation, but it's improved with the further incorporations up to the optimum condition at $15 \%(\mathrm{w} / \mathrm{w})$ [EMIm]Ac ionic liquid. After that condition, a decreasing of tensile strength is happened. The optimum tensile strength is at $15 \%(\mathrm{w} / \mathrm{w})$ of [EMIm]Ac ionic liquid incorporation to $\mathrm{LiClO}_{4}$-complexed $\mathrm{MC}$, that is $24.19 \mathrm{MPa}$. From this data, it can be explained that presence of [EMIm]Ac ionic liquid has been succeeded to deconstruct interaction happened inter-chain methyl cellulose biopolymer in early its incorporation and after optimum condition.

Elongation break ( $\left.E_{\text {break }}\right)$ analysis was conducted to both before and after [EMIm]Ac ionic liquid incorporation to $\mathrm{LiClO}_{4}$-complexed methyl cellulose. For the various of [EMIm]Ac ionic liquid incorporation: $5,10,15,20,25$, and 30 $\%(\mathrm{w} / \mathrm{w})$ as plasticizers of BPE membranes: $\mathrm{MC} / \mathrm{LiClO}_{4} /[\mathrm{EMIm}] \mathrm{Ac}$ is illustrated in Figure 15. From the figure could be depicted the effect of [EMIm]Ac plasticization significantly increases the fabricated membrane strain up to incorporation of $30 \%$ weight of [EMIm]Ac ionic

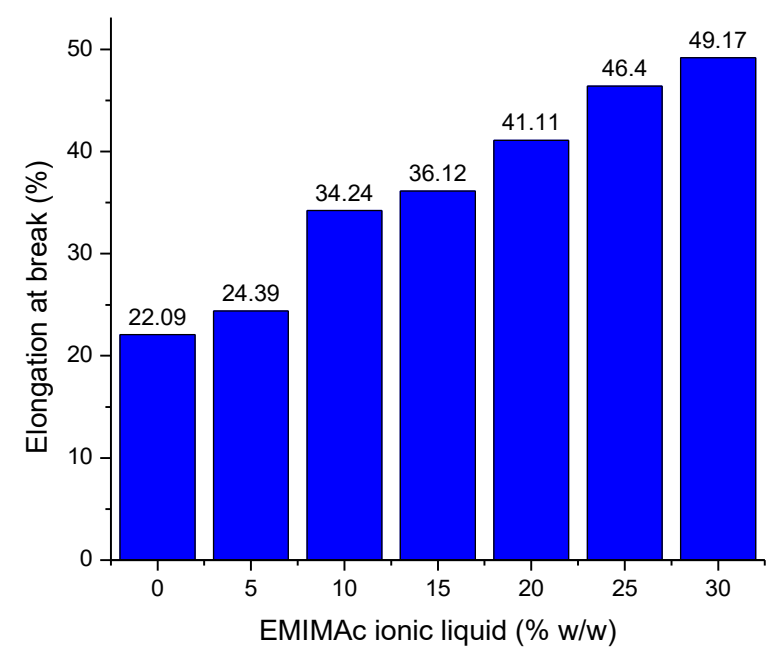

Figure 15. Elongation at break (Strain) of BPE Membranes 
liquids, and release $49.7 \%$ at high value. Increasing of elongation break ( $E_{\text {break }}$ ) is indicating biopolymer electrolyte membranes flexible properties. Mechanical properties with sufficient tensile strength and good elongation break indicate chain flexibility of biopolymer electrolyte that is able to support chain motions which contribute to Li-ion transport.

There is a mechanical properties difference between before and after optimum condition of tensile strength. Before the optimum condition, toughness increases, it means elongation at break increase as tensile strength increase. This property is predicted as a plastic behavior. After the optimum condition, the mechanical properties deformation is happened, where elongation at break decreases as tensile strength increases, this property called as an elastic-like behavior. It could be concluded that [EMIm]Ac ionic liquid incorporation to the MC

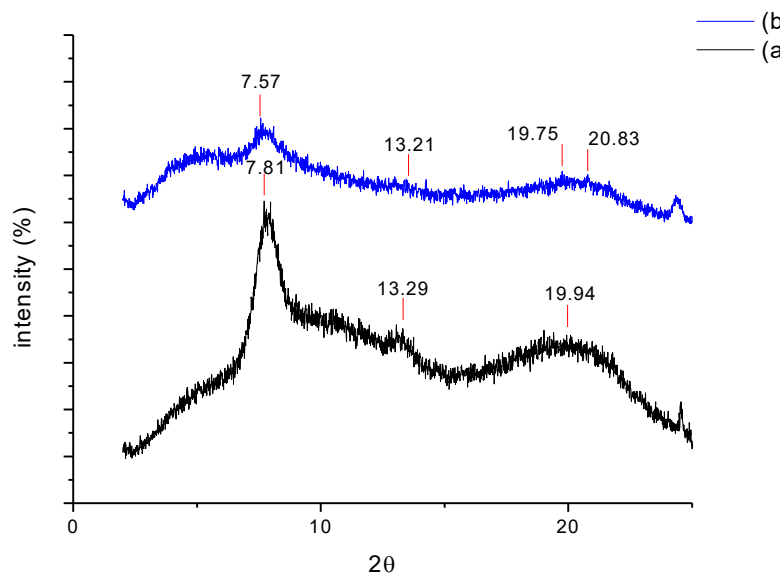

Figure 16. XRD pattern of Biopolymer electrolyte membranes, (a) $\mathrm{MC} / 10 \%$ (w/w) $\mathrm{LiClO}_{4}$; (b) $\mathrm{MC} / 10 \% \mathrm{LiClO}_{4} / 15 \%(\mathrm{w} / \mathrm{w})$ [EMIm] Ac

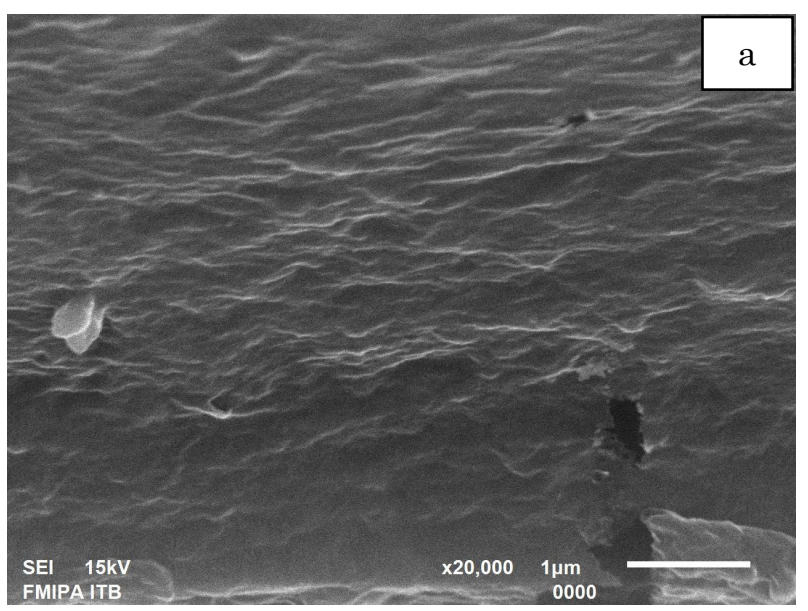

membranes gave plasticization and hardelastic effect significantly at various compositions.

3.8 Crystallinity Analysis of BiopolymerElectrolyte Membrane, BEM: MC/LiClO $4 /$ [EMIm]Ac

Some diffraction peaks of methyl cellulose are commonly at $\sim 7.8^{\circ} ; 13.4^{\circ} ; 20^{\circ}$, and $21^{\circ}$ [34] correlate to Miller indices of (001), (100), (012), (102). Quiroz et al. [35] said that methyl cellulose-based films release an amorphouscrystalline structure, especially a high intensity of $2 \theta=8^{\circ}$, a trymethylglucose type crystalline order and broad peak at $2 \theta=21.5^{\circ}$.

From Figure 16, diffraction peaks patterns of $\mathrm{LiClO}_{4}$-complexed methyl cellulose are compared to [EMIm]Ac-plasticized of $\mathrm{LiClO}_{4}$ complexed methyl cellulose. Both are showing Miller indices but having different intensity and a little bit of $2 \theta$-shifts. Presence of [EMIm]Ac ionic liquid weakens inter- and intramolecular hydrogen bonds, could be detected by decreasing intensity. Decreasing of peak intensity correlate to decreasing of crystallinity; vice versa, increasing of amorphous. Decreasing of crystallinity gives contribution to Li-ion migration and segmental motion of polymer chain (methyl cellulose in this case), so it can improve ionic conductivities.

\subsection{The Morphology Study using Scanning Electron Microscopy (SEM)}

Morphology studies was performed to both $\mathrm{MC} / 10 \% \mathrm{LiClO}_{4}$ and $\mathrm{MC} / 10 \% \mathrm{LiClO}_{4} / 15 \%$ [EMIm]Ac by Scanning Electron Microscopy (SEM) (depicted at Figure 17). There is a significant difference between both samples.

Figure 17. SEM analysis of (a) $\mathrm{MC} / 10 \% \mathrm{LiClO}_{4}$; (b) $\mathrm{MC} / 10 \% \mathrm{LiClO}_{4} / 15 \%$ [EMIm] Ac 
$\mathrm{LiClO}_{4}$-complexed methyl cellulose shows morphology with no pores, while [EMIm]Ac ionic liquid-plasticized of $\mathrm{LiClO}_{4}$-complexed methyl cellulose have some pores on its morphology.

There is a significant difference between both samples. $\mathrm{LiClO}_{4}$-complexed methyl cellulose shows morphology with no pores, while [EMIm]Ac ionic liquid-plasticized of $\mathrm{LiClO}_{4-}$ complexed methyl cellulose have some pores on its morphology. The presence of pores indicate decreasing of crystallinity index as effect of [EMIm]Ac ionic liquid plasticization. [EMIm]Ac ionic liquid incorporation is able to weaken hydrogen interaction intra-/inter chain of methyl cellulose as polymer host. Therefore, the [EMIm]Ac ionic liquid has been succeeded to role as spacer which contribute the Li-ion diffusion and subsequently exhibited the high ionic conductivity.

3.10 Thermal Analysis of Biopolymer Electrolyte Membranes, BPE Membrane: $\mathrm{MC} / \mathrm{LiClO}_{4} /$ [EMIm]Ac

Thermal stability of BPE: $\mathrm{MC} / \mathrm{LiClO}_{4} /[\mathrm{EMIm}] \mathrm{Ac}$ was studied by Thermogravimetry Analysis (TGA), Differential Thermal Analysis (DTA) and Difference Thermo Gravimetry (DTG). The thermogram below shows TG, DTG, and DTA data of $\mathrm{MC} / \mathrm{LiClO}_{4}$ (Figure 18a) and $\mathrm{MC} / \mathrm{LiClO}_{4} /[\mathrm{EMIm}] \mathrm{Ac}$ (Figure $18 b)$.

If we compare to previous report [6], the onset degradation temperature of methyl cellulose-based membrane at $\sim 300{ }^{\circ} \mathrm{C}$, a decreasing occurred of onset degradation temperature after $\mathrm{LiClO}_{4}$ salt incorporation to methyl cellulose. Figure 18a depicted the water removal

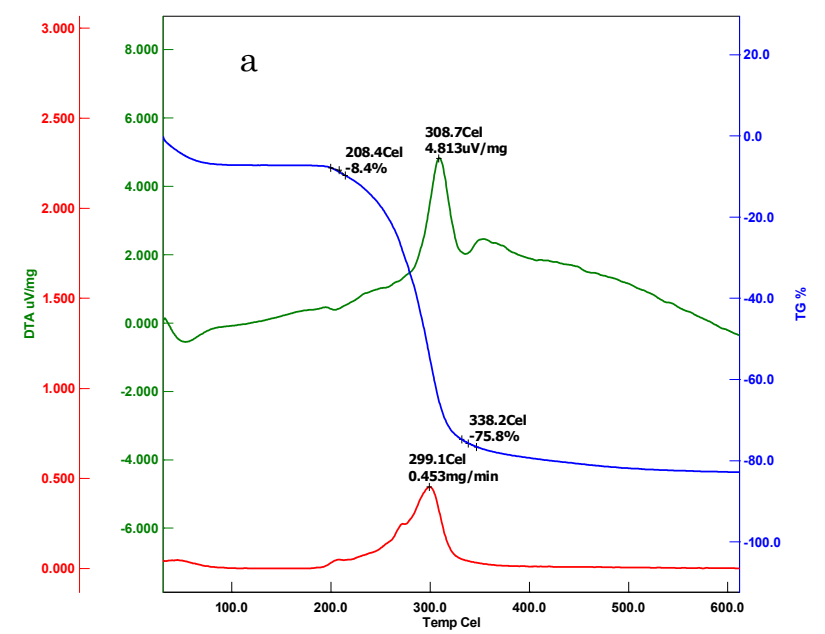

and the moisture desorption stages at $55.5{ }^{\circ} \mathrm{C}$, while the onset decomposition temperature was occurred at $208.4{ }^{\circ} \mathrm{C}$ with $8.45 \%$ weight loss, and with $0.453 \mathrm{mg} /$ minutes of rate at $299.1^{\circ} \mathrm{C}$, and melts at $308.7{ }^{\circ} \mathrm{C}$. The offset decomposition temperature was occurred at 338.2 ${ }^{\circ} \mathrm{C}$ and remaining $24.2 \%$ of weight loss. A decreasing of $\mathrm{LiClO}_{4}$-complexed methyl cellulose membrane onset decomposition temperature is attributed to crystallinity reducing as impacts disturb of inter-chain interaction of methyl cellulose. After the offset decomposition temperature of $\mathrm{LiClO}_{4}$-complexed methyl cellulose, the charred at $600{ }^{\circ} \mathrm{C}$ occurred and the final residue has $24 \%$ of weight.

[EMIm]Ac ionic liquid incorporation gives new reason of thermal properties. Basically, its introduction into $\mathrm{LiClO}_{4}$-complexed methyl cellulose matrix decreased the methyl cellulose inter-chain interactions, which contribute to Li-ion transport. Figure $18 \mathrm{~b}$ depicted an increasing of onset decomposition temperature at $255.9{ }^{\circ} \mathrm{C}$ with $4.1 \%$ of weight loss, and with $0.355 \mathrm{mg}$ of rate at $319.4{ }^{\circ} \mathrm{C}$, due to the [EMIm]Ac ionic liquid incorporation. The offset decomposition temperature was occurred at $338.2{ }^{\circ} \mathrm{C}$ and remaining $24.2 \%$ of weight loss. The offset decomposition temperature is occurred at $369.6{ }^{\circ} \mathrm{C}$ and remaining $20.9 \%$ of weight loss. After the offset decomposition temperature of [EMIm]Ac-plasticized $\mathrm{LiClO}_{4}$ complexed methyl cellulose, charred at $600{ }^{\circ} \mathrm{C}$ happened and remains $20.9 \%$ weight of residue. Although the crystallinity properties decrease, but the [EMIm]Ac ionic liquid acts to increase inter-molecular interaction (polymer chain and [EMIm]Ac ionic liquid), exhibits new thermal properties which is different to the

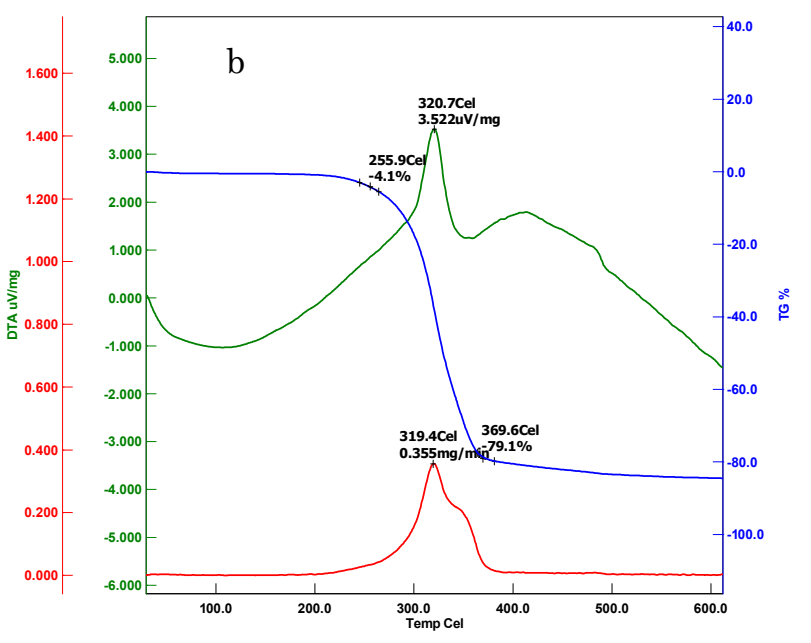

Figure 18. Thermogram of biopolymer electrolyte membranes, (a) $\mathrm{MC} / 10 \%$ (w/w) $\mathrm{LiClO}_{4}$; (b) $\mathrm{MC} / 10 \%$ $\mathrm{LiClO}_{4} / 15 \%$ (w/w) [EMIm]Ac 
usual deconstruction of lithium salts to membrane matrix. This fact showed that the [EMIm]Ac ionic liquid is able to improve the biopolymer electrolytes thermal properties.

\section{Conclusions}

The 1-ethyl-3-metylimidazolium bromide, [EMIm]Br ionic liquid, as precursor of [EMIm]Ac, has been successfully synthesized from 1-methyl imidazole and bromoethane using Microwave Assisted Organic Synthesis (MAOS) Method, at power of 100 watts, $50{ }^{\circ} \mathrm{C}$, 15 minutes and medium stirring. The 1-ethyl3-metylimidazolium acetate, [EMIm]Ac ionic liquid, has been successfully synthesized from metathesis reaction between [EMIm]Br ionic liquid and potassium acetate $\left(\mathrm{CH}_{3} \mathrm{COOK}\right)$ at room temperature for 1 hour. The [EMIm]Ac ionic liquid has the effect to increase the biopolymer electrolyte membranes of $\mathrm{LiClO}_{4-}$ complexed methyl cellulose, and at $15 \%$ [EMIm]Ac ionic liquid exhibited the optimum results in ionic conductivity, tensile strength, elongation break and thermal stability properties, which are $9.16 \times 10^{-3} \mathrm{~S} . c m^{-1}, 24.19 \mathrm{MPa}$, $36.43 \%, \sim 256$ and $\sim 370{ }^{\circ} \mathrm{C}$, respectively. These performances are sufficient to be used as a $\mathrm{Li}$ ion batteries separator candidate.

\section{Acknowledgment}

This work was supported by Chemistry Department, Faculty of Mathematics and Natural Sciences, Institut Teknologi Bandung (ITB), Bandung, Indonesia. The authors are also thankful to all of technician for data analysis and measurement in Analytical Laboratory (ITB), Testing Laboratory of Textile Center (Ministry of Industry Affairs of Republic of Indonesia), Hydrogeology and Hydrogeochemistry Laboratory (Department of Mining Engineering, ITB), Basic Science Centre-A (ITB), Integrated Laboratory and Technology Innovation Center (University of Lampung), respectively.

\section{References}

[1] Bolloli, M., Antonelli, C., Molmeret, Y., Alloin, F., Iojoiu, C., Sanchez, J. (2016). Nanocompostie poly(vynilidine fluoride) / nanocrystalline cellulose porous membranes as separators for lithium-ion batteries. Electrochemica Acta, 214: 38-48.

[2] Li, M., Wang, X., Yang, Y., Chang, Z., Wu, Y., Holze, R. (2015). A dense cellulose-based membrane as a renewable host for gel poly- mer electrolyte of lithium ion batteries. Journal of Membrane Science, 476: 112-118.

[3] Polu, A., Rhee, H. (2017). Ionic liquid doped PEO-based solid polymer electrolytes for Lithium-ion polymer batteries. Int. J. Hydrogen Energy, 42: 7212-7219.

[4] Cheng, D., Yang, X., He, Z., Ni, Y. (2016). Potential of cellulose-based materials for lithium-ion batteries (LIB) separator membranes. Journal of Bioresources and Bioproducts, 1(1): 18-21.

[5] Mindemark, J., Sun, B., Torma, E., Brandell, D. (2015). High-performance solid polymer electrolytes for lithium batteries operational at ambient temperature. Journal of Power Sources, 298: 166-170.

[6] Xiao, S. Y., Yang, Y. Q., Li, M. X., Wang, F. X., Chang, Z., Wu, Y. P., Liu, X. (2014). A composite membrane based on a biocompatible cellulose as a host of gel polymer electrolyte for lithium ion batteries. Journal of Power Sources, 270: 53-58.

[7] Jabbour, L., Bongiovanni, R., Chaussy, D., Gerbaldi, C., Beneventi, D. (2013). Cellulosebased Li-ion batteries: a review. Cellulose, 20: 1523-1545.

[8] Bruce, P. (1995). Structure and electrochemistry of Polymer Electrolytes. Electrochemica Acta, 40: 2077-2086.

[9] Bergman, R., Brodin, A., Engberg, D., Lu, Q., Angell, A., Torell, L. (1995). Fast and slow relaxation processes in polymer electrolytes. Electrochemica Acta, 40: 2049-2055.

[10] Liew, C., Ramesh, S. (2015). Electrical, structural, thermal and electrochemical properties of corn strarch-based biopolymer electrolytes. Carbohydrate Polymers, 124: 222-228.

[11] Liang, B., Jiang, Q., Tang, S., Li, S., Chen, X. (2015). Porous polymer electrolytes with high ionic conductivity and good mechanichal property for rechargeable batteries. Journal of Power Sources, 307: 320-32.

[12] Doyle, R., Chen, X., Macrae, M., Srungavarapu, A., Smith, L., Gopinadhan, M. O., Focil, S. (2014). Poly(ethylenimine-based polymer blends as single-ion lithium conductor. Macromolecules, 47: 3401-3408.

[13] Kumar, Y., Hashmi, S., Pandey, G. (2011). Lithium ion transport and ion-polymer interaction in PEO based polymer electrolyte plasticized with ionic liquid. Solid State Ionics, 201: 73-80.

[14] Xiong, M., Tang, H., Wang, Y., Pan, M. (2014). Ethylcellulose-coated polyolefin separators for lithium-ion batteries with improved safety performance. Carbohydrate Polymers, 101: 1140-1146. 
[15] Qiu, L., Shao, Z., Wang, D., Wang, F., Wang, W., Wang, J. (2014). Novel polymer Li-ion binder carboxymethyl cellulose derivative enhanced electrochemical performance for Li-ion batteries. Carbohydrate Polymers, 112: 532538 .

[16] Samsudin, A., Kuan, E., Isa, M. (2011). Investigation of the potential of proton-conducting biopolymer electrolytes based methyl cellulose-gycolic acid. International Journal of Polymer Analysis and Characterization 16(7): 477-485.

[17] Saha, N., Sarkar, G., Roy, I., Rana, D., Bhattacharyya, A., Mukhopadhyay, A., Chattopadhyay, D. (2016). Studies on methylcellulose/pectin/montmorillonite nanocomposite films and their application possibilities. Carbohydrate Polymers, 136: 1218-1227.

[18] Pandian, S., Raju, S., Hariharan, K., Kolake, S., Park, D., Lee, M. (2015). Fuctionalized ionic liquids as electrolytes for lithium-ion batteries. Journal of Power Sources, 286: 204209.

[19] Yuen, X., Cheng, G. (2015). From cellulose fibrils to single chains: understanding cellulose dissolution in ionic liquids. Phys. Chem. Chem. Phys., 17: 31592-31607.

[20] Isik, M., Sardon, H., Mecerreyes, D. (2014). Ionic liquids and cellulose: Dissolution, chemical modification and preparation of new cellosic materials. International Journal Molecular Sciences. 15: 11922-11940.

[21] Lewandowski, A., Mocek, A. (2009). Ionic liquids as electrolytes for Li-ion batteries-An overview of electrochemical studies. Journal of Power Sources, 194: 601-609.

[22] Pagot, G., Bertasi, F., Vezzu, K., Nawn, G., Pace, G., Nale, A., Noto, V. (2018). Correlation between properties dan conductivity mechanism in Poly(vinyl alcohol)-based lithium solid electrolytes. Solid State Ionics, 320: 177-185.

[23] Wasserscheid, P., Welton, T. (2002). Ionic Liquids in Synthesis. Wiley-VCH.

[24] Rana, K., Rana, S. (2014). Microwave Reactors: A Brief Review on Its Fundamental Aspect and Applications. Open Access Library Journal, 1: 1-20.

[25] Asikkala, J. (2008). Application of Ionic Liquids and microwave activation in selected organic reaction. PhD Dissertation. Department of Chemistry, University of Helsinki.
[26] Ratti, R. (2014). Ionic liquids: Synthesis and Applications in catalysis. Advances in Chemistry, 2014: 1-16.

[27] Surati, M., Jauhari, S., Desai, K. (2012). A brief review: Microwave assited organic reaction. Archives of Applied Science Research, 4: 645-661.

[28] Ohtsuki, J., Matsumoto, K., Hagiwara, R. (2009). Physical and Electrochemical Properties of 1-ethyl-3-methylimidazolium Ionic Liquids of Mixed Anions, (FH)nF- , BF4- , and $\mathrm{N}\left(\mathrm{SO}_{2} \mathrm{CF}_{3}\right)^{2-}$. Electrochemistry, 8: 624-626.

[29] Chaurasia, S., Singh, R., Chandra, S. (2011). Dielectric relaxation and conductivity studies on $\left(\mathrm{PEO}: \mathrm{LIClO}_{4}\right)$ Polymer Electrolyte with Added Ionic Liquid [BMIM][PF6]: Evidence of Ion-ion Interaction. Polymer Physics, 49: 291300.

[30] Farran, A., Cai, C., Sandoval, M., Xu, Y., Liu, J., Hernaiz, M., Linhardt, R. (2015). Green solvents in carbohydrate chemistry: From raw materials to fine chemicals. Chemical Reviews, 115(14): 6811-6853.

[31] Brandt, A., Grasvik, J., Hallett, J., Welton. (2013). Desconstruction of lignocellulosic biomass with ionic liquids. Green Chemistry, 20: 550-583.

[32] Rani, M., Rudhziah, S., Ahmad, A., Mohamed, N. (2014). Biopolymer electrolyte based on derivatives of cellulose from kenaf bast fiber. Polymers, 6: 2371-2385.

[33] Mobarak, N., Jumaah, F., Ghani, M., Abdullah, M., Ahmad, A. (2015). Carboxymethyl carageenan based biopolymer electrolytes. Electrochemica Acta, 175: 224-231.

[34] Orasugh, J., Saha, N., Sarkar, G., Rana, D., Mishra, R., Mondal, D., Chattopadhyay, D. (2018). Synthesis of methylcellulose/cellulose nano-crystals nanocomposites: Material properties and study of sustained release of ketorolac tromethamine. Carbohydrate Polymer, 188: 168-180.

[35] Quiroz, M., Lecot, J., Bertola, N., Pinotti, A. (2013). Stability of methylcellulose-based films after being subjected to different conservation and processing temperatures. Materials Science and Engineering C, 33: 2918-2925. 